Afrika Focus, Vol.8, Nr.1, 1992,pp 5-32

\title{
LES ASPECTS PRODUCTIFS DE L'ÉCONOMIE INFORMELLE. RECHERCHE DES INDICATEURS POUR UNE RÉPONSE AU DÉVELOPPEMENT EN AFRIQUE
}

\section{Bagalwa Basemake Gaspard MUHEME}

Julius De Geyterstraat 24

$B-2660$ HOBOKEN (BELGIQUE)

CHAMPS D'INVESTIGATION: économie informelle dans les Pays en Voie de Développement

\section{SUMMARY}

\section{PRODUCTIVE ASPECTS OF THE INFORMAL ECO- NOMY. RESEARCH INTO THE INDICATORS FOR AN ANSWER TO THE DEVELOPMENT OF AFRICA}

This article aims at deepening our understanding of the concept informal economy. It is the case that definitions with regard to the reality of this phenomenon vary in function of the author and the countries studied. Indeed, the concept needs to be discussed right across the countries of the West, the East European countries under the old system, i.e. until 1990, and the countries of Africa.

With regard to the informal economy in Africa, the characteristics of irrationality and the lack of initiative on the part of economic agents has often been posited. However, this economic phenomenon is neither exclusive to Africa nor is it confined to the present time. Only a comparative approach will enable one to arrive at some precision with regard to the concepts used in the economic literature.

Our primary goal is to place this informal economy within the global perspective of African development. This informal economy sets itself up as a creative attempt, 
often able to give efficient answers, whether in the field of agriculture or in other forms of activity, to the challenges of badly mismanaged societies.

The article rests on a number of key-terms:

informal economy; plural activities; integrative economy; petty commodity production and consumption; interdependency of sectors.

KEY WORDS: informal economy, plural activities, integrative economy, petty commodity production and consumption, interdependency of sectors

\section{INTRODUCTION."}

La réalité du phénomène d'économie informelle connaît depuis les années 1970 un retentissement. Pourtant ni le substantif en soi ni son qualificatif ne sont des mots nouveaux. La question est préoccupante depuis des années (1). En effet si l'on remonte au XVIème siècle où se situe le projet financier de Lorenzo TONTINI (2), il se dessine une économie basée sur la tontine. De l'avis des économistes, des recherches se poursuivraient, depuis l'an 1877 avec LEROY-BEAULIEU, autour de l'évasion et de la fraude fiscales. Les études reconnaissent ainsi une économie non officielle en Finances Publiques. Un certain nombre d'idées associées à l'économie informelle ne seraient donc pas neuves. Comme l'affirme Xavier GREFFE (3) avec l'économie de l'autogestion, les idées liées à l'informel se trouvent déjà dans la pensée les premiers théoriciens du mouvement coopératif. Selon qu'il s'agit d'activités économiques dissimulées aux autorités, dans tous les pays et à toutes les époques, des personnes essayent de pratiquer de l'économie informelle (4). Bref, le fait d'être en vogue dès les années 1970 ne suffit pas pour nier l'existence antérieure de l'informel.

Pour Alfred SAUVY (1980), le concept est aussi vieux que la loi. Par contre, Bruno LANVIN (1981) réservera l'expression aux activités de débrouillardise dans les pays non- industrialisés. Pierre PESTIEAU (1989) paraît emboîter le même pas en distinguant un secteur monétisé dans toutes ses fonctions d'un secteur non

- Ce texte a été réalisé dans le cadre d'une thèse de doctorat en préparation a la Faculté des Sciences économiques, sociales et politiques de l'UCL sous la direction du Professeur Michel NORRO. L'auteur tient à remercier le Prof. M. NORRO pour ses avis éclairés. 
monétisé selon les principes de l'économie de subsistance et du troc. Un tel point de vue amène le Professeur PESTIEAU à taxer d'"économie informelle" ses équivalents des pays en développement et de "seconde économie" ceux des pays socialistes (5). On pourrait s'interroger sur les racines d'une telle catégorisation. En effet, on peut se demander si une modification du concept selon les pays concernés ne relèverait pas de l'arbitraire et ne susciterait pas des options idéologiques. Certes, des spécificités peuvent se dessiner selon les législations propres aux pays.

Cependant, pour plus de clarté, il vaut mieux cerner d'abord les supports d'une telle répartition au niveau de l'ensemble des activités économiques. Si la typologie au niveau interne de l'économie se justifie, la variété de termes synonymes selon les pays concernés s'avère, d'un point de vue de l'analyse économique, anodine et relèverait même d'un excellent jeu de mots. La distinction apparemment marquée entre parallele, souterraine et informelle serait-elle dépourvue d'ambiguïté? La même remarque ne vaudrait-elle pas pour les frontières tracées, dans certaines approches, entre le légal et l'illégal même si ces dernières peuvent encore être déterminées par l'environnement socio-économique et historo-culturel?

Toutefois, la désignation de l'économie informelle se poursuit, par exemple, à travers les expressions de production au-delà des normes prescrites par la réglementation, d'argent secret (Ingo WALTER, 1985), d'argent noir (Pierre PEAN, 1988), de point d'appui pour minimiser les coûts et de secteur non structuré ouvert ou fermé (Jacques CHARMES, 1983), d'économie sociale (Stuart HENRY, 1982) ou encore d'activités productives ou non-productives non évaluées par les comptes nationaux (Jean-Charles WILLARD, 1990).

Ces notions permettent de relativiser le qualificatif du phénomène complexe et multiforme regroupé provisoirement pour notre part, sous le nom d'économie informelle. Etant donné la multiplicité des caractéristiques, nous venons d'apporter quelque précision quant à sa désignation dans la littérature économique. Ensuite, il nous faudra citer des exemples, parmi beaucoup d'autres, illustrant ce que peut être l'économie informelle dans un pays industrialisé, qu'il soit de l'Ouest ou de l'Est, et dans les pays africains.

Enfin, nous essayerons de contribuer, à l'issue des exemples, à déterminer le concept à approfondir pour une réponse au développement de l'Afrique. 

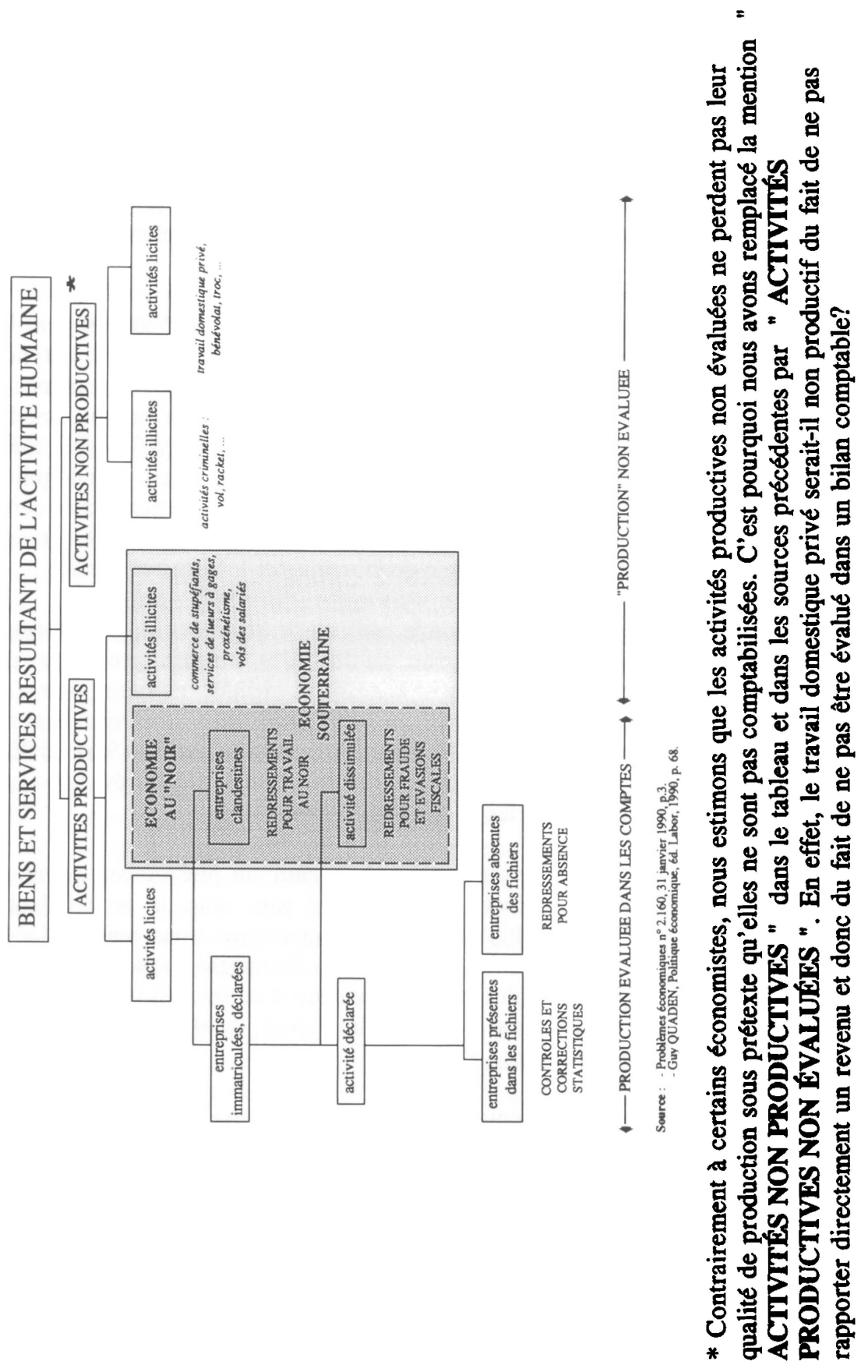


\section{LES EXEMPLES D'ÉCONOMIE INFORMELLE DANS LES PAYS INDUSTRIALISÉS.}

\subsection{En Occident: les activités informelles licites et illicites.}

Observée d'Europe occidentale (6) ou d'Amérique du Nord, l'économie informelle regroupe des productions, des revenus, des échanges et même des dons non déclarés à une autorité fiscale quelconque pour des motifs frauduleux ou tout à fait légaux. Certains procurent un avantage financier, d'autres n'en procurent pas du tout. Ivan ILLICH (7) retient, de son séjour américain, quelques spécimens: les travaux agricoles saisonniers, la vendange ou la récolte d'agrumes, par exemple, effectués par des Mexicains aux Etats-Unis. L'analyse qu'on en fait ne s'éloigne pas de la réalité. Citons, dans ce même ordre d'idée, les échanges de bons procédés tels qu'entretenir la pelouse d'un avocat. En échange, ce défenseur veille à ce que votre maison bâtie sans permis de construire, échappe à l'inspection de l'administration. Le garagiste remplace le carburateur sur la voiture du comptable, lequel de son côté, lui remplit ses déclarations d'impôts. Voilà des exemples, parmi beaucoup d'autres, illustrant ce que peut être le secteur informel dans un pays occidental.

En Europe Occidentale, une mère assure la garde d'enfants à la maison ou elle accomplit d'autres travaux au ménage pour suppléer au budget du foyer. Elle n'est pas déclarée à l'Etat. Son travail est dit clandestin et presque réprimé suivant des limites tracées par les pays, les communes et même suivant que l'activité est exercée pour soi-même ou pour autrui. Dans le cas où ce travail échappe aux projecteurs économiques, il relève de l'économie informelle.

Certains, comme Marie-Annick BARTHE, se plairont de l'appeler "économie cachée" (8) ou encore "travail fantôme" d'après Ivan ILLICH qui en a écrit tout un livre. Pour autant que cette économie repose sur des motifs frauduleux, Pierre ROSANVALLON (1980), la taxera d' "occulte" et de "domestique" si elle est parfaitement licite.

Toutefois l'essentiel ne devrait pas consister, nous semble-t-il, à situer l'activité exercée pour soi ou pour autrui par rapport à la légalité ou à l'illégalité. Il conviendrait, d'après un schéma forgé par André GORZ (1988) (9) d'asseoir une certaine rationalité. Bref, situer l'activité informelle soit par rapport à sa production soit par rapport à son but d'échange marchand. 
Tableau 2

\begin{tabular}{|l|c|c|c|c|c|c|c|}
\hline $\begin{array}{c}\text { Critéré } \\
\text { Secteur }\end{array}$ & $\begin{array}{c}\text { Qualifi- } \\
\text { cation }\end{array}$ & Porte & $\begin{array}{c}\text { Ressour- } \\
\text { ces }\end{array}$ & $\begin{array}{c}\text { Pro- } \\
\text { priété }\end{array}$ & $\begin{array}{c}\text { Produc- } \\
\text { tion }\end{array}$ & $\begin{array}{c}\text { Techni- } \\
\text { que }\end{array}$ & Marché \\
\hline $\begin{array}{l}\text { Secteur } \\
\text { moderne } \\
\text { (FORMEL) }\end{array}$ & $\begin{array}{c}\text { scolaire } \\
\text { ou } \\
\text { expéri- } \\
\text { ence }\end{array}$ & fermée & $\begin{array}{c}\text { impor- } \\
\text { tées } \\
\text { capita- } \\
\text { listiques }\end{array}$ & $\begin{array}{c}\text { action- } \\
\text { nariat }\end{array}$ & grande & lourde & protégé \\
\hline $\begin{array}{l}\text { Secteur } \\
\text { non } \\
\text { struc- } \\
\text { turé } \\
\text { (INFOR- } \\
\text { MEL) }\end{array}$ & $\begin{array}{c}\text { tissage } \\
\text { extra- } \\
\text { scolaire }\end{array}$ & ouverte & $\begin{array}{c}\text { locales } \\
\text { faible } \\
\text { capital }\end{array}$ & familiale & petite & $\begin{array}{c}\text { légère et } \\
\text { main- } \\
\text { d'oeuvre } \\
\text { abon- } \\
\text { dante } \\
\text { ministré } \\
\text { et } \\
\text { concur- } \\
\text { rentiel }\end{array}$ \\
\hline
\end{tabular}


Ainsi l'léconomie retiendrait deux champs économiques distincts:

a. la sphère d'activités marchandes fondée sur la valeur d'usage, l'échange marchand, l'identité (publique, privée ou non motivée) et la mesure du temps et du rendement;

b. la sphère d'activités non marchandes regroupant le travail pour soi (auto-entretien, services de proximité, entraide entre voisins), la sphère micro-sociale ou domestique et les activités "autonomes" (10), c'est à dire sans utilité économique.

L'économie informelle n'est pas seulement illicite. En dehors des activités de "cumulards" dans les pays à forte pression fiscale et parafiscale comme les pays scandinaves, outre le troc des services aux Etats-Unis d'Amérique et en dehors du travail accompli sans facture, ou avec facture biaisée par un agent déclaré en France par exemple, il existe des initiatives de citoyens. Tel est le cas des expériences des "Burgerinitiativen" (11) en Allemagne. Ces initiatives dites alternatives (santé, école, 3ème âge, handicapés physiques et mentaux, jeunes marginaux ou non, drogue, alcoolisme, solitude, mères célibataires, le travail des enfants etc.) fonctionnent comme activités non déclarées destinées à assurer la survie d'une mouvance menacée par les interdits professionnels. Elles relèvent des activités informelles non marchandes, régies par des programmes économiques d'assistance directe (les organisations de base) ou d'assistance indirecte (les organisations de solidarité internationale). A propos de l'économie informelle dans les pays industrialisés, l'impression générale répandue par de nombreuses études est celle d'une économie illicite fondée sur l'évasion et la fraude fiscales, le crime et la drogue ou les revenus de la corruption (12). Les analyses font très peu d'élaboration sur le DIY work (le Do It Yourself) britannique et sur le "social informal economy". Pourtant l'on assiste a la multiplication des centres de bricolages dans les pays industrialisés.

Au delà de la synthèse issue d'André GORZ, trois études (13) semblent, à ce sujet, sortir de l'ordinaire: Stuart HENRY (1982), Stephen SMITH et Suzanne WIED-NEBBELING (1986) et, Jean-Charles WILLARD (1990). Leur façon de regrouper l'ensemble des activités économiques permet de distinguer les nomenclatures de l'économie informelle par rapport à l'économie officielle. Le tableau 2 en établit une synthèse.

Il ressort de cet ensemble des Biens et Services résultant de l'activité socio-économique, des sous-ensembles d'activités complémentaires et différents. 
En effet les activités productives, évaluées ou non y compris leurs Programmes de Gestion c'est à dire les Organisations font, toutes, partie d'un système économique. Evaluées ou non dans les comptes, leur modalité de fonctionnement n'est pas sans impact sur l'ensemble de la production nationale.

Ainsi, le système économique tient sur quatre bases:

a. les activités productives de l'économie officielle, elles constituent les comptes nationaux;

b. les activités productives de l'économie informelle: elles sont absentes des comptes nationaux soit par convention (Angleterre, Allemagne), soit par manque de moyen de les inclure car elles sont souterraines, irrégulières ou dissimulées;

c. les activités évaluées de l'économie informelle: elles sont comptabilisables mais, dans la mesure où elles sont criminelles, elles échappent au fisc et au statisticien;

d. les activités du Do It Yourself (le DIY) reconnues mais non évaluées: l'économie informelle sociale et le secteur autonome.

\subsection{En Europe de l'Est et en Union soviétique sous l'ancien régime ou jusqu'en 1990.}

A propos du secteur informel occidental, la situation de l'ensemble des Biens et Services de l'activité humaine permet d'établir ce constat: l'économie parallèle est inséparable de l'économie officielle étant donné l'imbrication du jeu économique de leurs rapports.

Toutes les économies se soudent à l'intérieur d'un même système économique. Même le secteur dit autonome n'est pas coupé de l'ensemble de la réalité socio-économique d'un pays. Il n'en va pas autrement de la seconde économie soviétique. Certaines théories limitent l'informel soviétique à "l'intérêt privé marqué par le gain" (14) et opposé avec la centralisation du système.

Dans cette optique, les activités informelles découlent de l'intrusion du secteur privé dans le secteur socialiste. Elles englobent la production effectuée en dehors de l'Etat d'une part et d'autre part la redistribution opérée au niveau de la population (15) sans un contrôle direct des planificateurs, des décideurs et des administrateurs (16). 
L'économie parallèle renferme différents aspects dont la production de lopins de terre familiaux, les transferts et redistribution cachés issus de l'activité professionnelle, la location privée des locaux d'habitation y compris les intérêts et commissions payés lors des opérations effectuées en dehors des canaux officiels. Cette économie renferme également le secteur privé autorisé et des formes de clandestinité entretenues par des travailleurs qui ménagent leur force de travail pour en faire un usage plus lucratif en dehors des heures réglementaires.

Ainsi O.V. OSIPENKO et I.G. KOZLOV (1989) distinguent trois versions de ce phénomène économique (17):

- la première version retient le travail individuel non réglementé par l'administration de l'Etat, la distribution de biens matériels et la spéculation. Bref, une économie privée illégale;

- la deuxième version est celle de la production de biens matériels et de services. Bien qu'officielle, cette production échappe aux comptes nationaux. Elle relève de l'économie publique illégale;

- la troisième version concerne les activités privées ou même les usines secrètes qui tournent à l'intérieur des entreprises de l'Etat. Ce phénomène correspond à la nature économique du socialisme tout en restant caché et donc à l'économie privée légale mais suspecte.

Ces économies seraient issues de l'écart entre la pratique économique et l'idéal fixé par les programmes politiques et sociaux. L'intérêt privé et l'intérêt collectif tendent ainsi à correspondre. Les cas de la Hongrie, de la Pologne et de la Yougoslavie, avant le vent de l'Est, semblent se prêter également à cette approche soviétique du secteur informel.

Par exemple, l'argent acquis par des voies criminelles tourne à la création d'une coopérative. Vous devez apporter au médecin d'Etat un poulet si vous voulez qu'il s'intéresse à votre cas, écrit Ivan ILLICH (18). Vous devez apporter des oeufs au fonctionnaire de l'état civil chargé de la publication des bans de mariage, poursuit l'auteur de Le travail fantôme. Voilà quelques transactions entre agents de l'économie apparente.

Finalement, du point de vue du concept (19), l'économie informelle soviétique serait une tentative de révolte contre la socialisation jugée abusive par l'individu, un effort de défendre ses droits en milieu économique. Ces tentatives ont comme causes la rareté des biens et services due à l'offre officielle inférieure à la demande. 


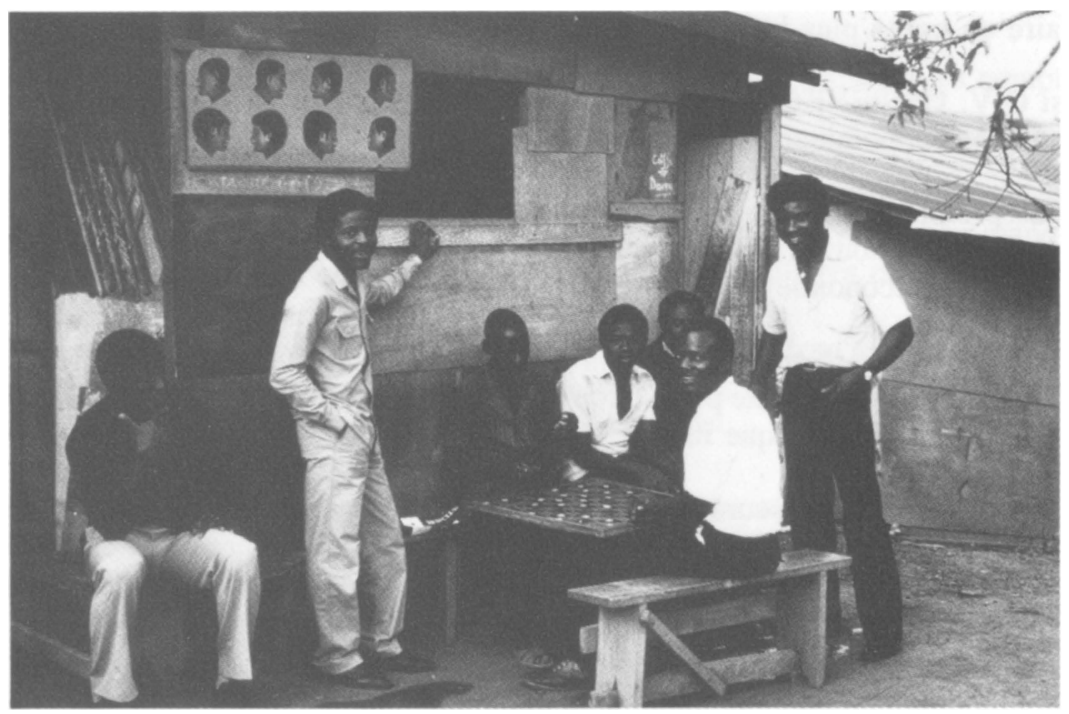

Un coiffeur au Cameroun.

Les petites activités socio-économiques visibles de la rue étaient les premieres d̀ être classées dans le secteur informel

D. Beke $^{\circ}$ 


\section{LES EXEMPLES D'ÉCONOMIE INFORMELLE DANS LES PAYS EN DÉVELOPPEMENT, SPECIALEMENT AFRICAINS.}

\subsection{L'état de la question dans la littérature.}

Certaines recherches (20) sur les petites activités économiques répandent l'idée de passage "de l'indigène à l'informel" (Marc LE PAPE, 1983). Selon cette vision, déjà en 1955, les débats adoptent avec Georges BALANDIER les notions de prolifération et de multiplication de petites activités.

Même les travaux de l'East Africa Royal Commission 1953-1955, et par la suite, la Conférence de Kericho (21) auraient abordé le secteur informel en milieu rural et urbain sous le spectre du chômage déguisé. L'expression "chômage déguisé" est entendue dans le sens de R. NURKSE. En effet des individus travaillant dans le petit artisanat représentent une vaste gamme de petits échanges par troc, de petites exploitations familiales ou sociétaires. Ils assurent péniblement leur existence. Ils vivent d'une fraction du revenu réel de quelques membres de leur famille. Le parasitisme sévit dans la mesure où des non-travailleurs vivent à la charge du salarié. Des transferts de la campagne vers la ville sous forme d'aides familiales s'opèrent. Le salariat à temps partiel, le quasi-salariat, le métayage urbain et la pluri-activité s'intensifient. Tels sont les approches du secteur informel dans le contexte africain entre 1960 et 1970 (22).

La théorie économique du développement, dans son évolution, permettra d'établir un classement (23) et adoptera à partir des travaux du Bureau International du Travail (1972) ce terme utilisé par Keith HART (1971) à savoir le "Secteur informel urbain". Pour le BIT, estime Lisa PEATTIE (1980: 23), le secteur informel, dans son rôle positif, est une branche de l'économie et du système urbain malgré sa faible productivité par rapport au secteur moderne. L'attention porte sur l'existence d'un vaste spectre de l'activité économique à caractère varié. Théoriquement, les activités du secteur informel sont sous-estimées et au niveau statistique, sous-évaluées (24).

Mais au-delà de la définition du BIT et des sous-ensembles composés de réseaux intégrés de production et de commercialisation liés parfois aux villages ruraux, aux cités urbaines et à l'industrie, le secteur informel englobe, d'un point de vue socio-anthropologique (J.F. LAE, 1990: 23-37), différentes logiques sociales à l'oeuvre dans la pratique de redistribution et de socialisation (25). Et par rapport à la faillite de l'Etat, le secteur informel incarnerait diverses tentatives d'inspiration 
sociale permettant de corriger les vices du système de l'Etat. Le flou dans lequel nage la taxonomie (26) établie par Jocelyne GAUDIN et Michel SCHIRAY (1984: 691-731) permet de reconnaître la complexité du concept. Du reste, la classification peu rigide présentée et dénoncée à la fois par Ignacy SACHS (1984: 110-111) contribue à avouer les limites et les imprécisions des recherches, quant aux critères du secteur informel, au cours des années 1970 et, dans une certaine mesure, les années 1980.

\subsubsection{Dans les années 1970.}

Le secteur informel se traduit par "le développement de petites activités permettant aux migrants ruraux et aux citadins n'ayant pu avoir accès aux emplois du secteur moderne de vivre et de survivre" (J. CHARMES, 1990: 11). En dépit de leur contribution à réduire les poches de sous-emploi, les activités informelles restent marginales et secondaires. Ces questions de résorption du chômage, de lutte contre la pauvreté et de satisfaction de besoins de base soulèvent tout le problème des exclus du système dominant de l'économie. Par rapport à ces absents du système, le secteur informel apparaît comme une réserve de main-d'oeuvre dans laquelle le secteur moderne puise la force de travail débordant des réservoirs ruraux. Ce thème de l'exclusion sera au coeur des débats sur l'économie informelle surtout face au dualisme et à l'intégration.

Avec le progrès de l'industrialisation, le secteur informel serait considéré comme activité antinomique du progrès (27). La littérature retient des critères: tout ce qui n'entre pas dans les structures institutionnelles légales de l'activité économique est considéré comme "informel". Il serait parasite de l'économie structurée.

Certaines études sur les critères du phénomène associent à l'informel toute activité non officielle ou toute économie domestique ayant ou n'ayant pas la monnaie comme intermédiaire de l'échange. Aussi l'informel ne serait pas mesurable au moyen des techniques économiques. Selon qu'il apparait ou qu'il échappe aux projecteurs économiques, l'informel serait légal ou illégal (28).

Bref, les considérations portées sur le phénomène du secteur informel accusent d'un flou où quelque chose semble se dessiner mais on ne le voit pas très bien. Le concept présente beaucoup d'ambiguité: il est associé à la marginalité tout en servant de poche d'emploi; il serait une économie de pauvreté tout en valorisant ses acteurs; bref, une confusion règne sur l'informel quand on ne découvre pas sa réalité de petite production. 


\subsubsection{Dans les années 80 .}

Par la suite, les autorités politiques tendent à reconnaître l'existence d'un secteur informel issu de leur laxisme, de leur répression mais aussi de leur paternalisme. Il se développe une économie dont l'aspect productif s'affirme avec le concept de "non structuré moderne" c'est-à-dire produisant des biens et services identiques à ceux du secteur moderne.

Les années 80 connaissent plusieurs explications et diverses dimensions du secteur informel. Le concept reste préoccupant étant donné qu'il s'applique à tout ce qui échappe a l'économie régulière tout en répondant aux structures de la vie quotidienne. En 1988, A. CHANDAVARKAR (29) s'interroge et commente: "le secteur informel serait-il une boîte vide ou un concept fourre-tout"? Son analyse le conduira à la réflexion sur la notion de "balance des paiements" pour désigner le compte retraçant l'ensemble des opérations intervenues au cours d'une période donnée, entre un pays et l'extérieur. L'auteur souligne l'imprécision du concept de "balance des paiements". Mais, malgré tout, la Banque Mondiale l'utilise comme instrument de sa comptabilité. Donc l'expression est fonction de l'usage qu'on en fait. Et comme il en est ainsi, le secteur informel serait un concept portemanteau: il s'adapte au type de vêtement qu'on y suspend. Bref, le secteur informel est un concept dont la science peut user selon la branche à étudier, autrement dit un concept global abordable selon différents points de vue scientifiques.

Notre point de vue s'avère économique basé sur la branche de production et de commercialisation même s'il existe d'autres perceptions du secteur informel qu'il ne faudrait pas réduire à la dimension économique.

Quelques exemples tirés du quotidien au cours des années 80 et début des années 90 contribueront à asseoir cette diversité interne du secteur informel et de déterminer, selon les objectifs, le concept à approfondir pour une perspective d'avenir.

- Première explication: l'expérimentation sociale (30).

Des groupes ayant une emprise sur une situation relativement délimitée peuvent mener des actions pour apporter des solutions propres à leurs problèmes en modifiant leurs rapports avec l'Etat et les marchés. Ce sont de nouveaux styles de vie. Ils peuvent s'exprimer à travers des activités autonomes (X. GREFFE, 1982: 4) ou de nouvelles formes d'organisation de la production et de la vie associative. Au Zaïre, par exemple, l'indifférence des autorités politiques a suscité par-ci par-là des mécontents qui, dans l'armée comme dans la société civile, ne perçoivent plus leur salaire. Pour se protéger contre les razzias éventuels de ces insatisfaits, les 
populations se cotisent pour se prémunir contre ces "nécessiteux" malveillants. Certaines fêtes populaires et surtout des veillées funèbres sont souvent ouvertes à tous: on y boit, on y mange gratuitement et on y retrouve la chaleur humaine. Le libre accès aux services offerts constitue un élément de réponse aux personnes sans occupation et susceptibles, dans leur délinquance, de nuire à la société.

Dans le domaine de la production, les petits agriculteurs et les artisans prennent de plus en plus conscience qu'ils doivent travailler en entreprises groupées pour faire face aux aléas des prix.

- Deuxième explication: la marché noir dû aux excès des gouvernements (Guy SORMAN, 1987: 55).

Guy SORMAN décrit les conditions d'un entrepreneur argentin et qui ressemblent à celles d'un africain:

"A trente kilomètres de Buenos Aires, il n'y a pas de téléphone! Il aurait volontiers recouru d un système privé pour pallier la défaillance du secteur public, mais l'autorisation, lui en a été refusée. Son usine moderne est donc surmontée d'une haute antenne-radio, parfaitement illégale. Par cette radio, il communique avec un bureau qu'il a dâ spécialement installer dans la capitale pour que ses cadres puissent téléphoner" (31).

Voilà un secteur permettant de survivre à des excès gouvernementaux mais qui pousse à investir dans le marché noir.

- Troisième explication: un comportement financier qui enchaîne l'Etat.

La mauvaise gestion des budgets par les autorités engendre la fuite des capitaux. Sur tous les continents des sommes d'argent ont une tendance naturelle à quitter les pays mal gérés. Commerçants et investisseurs mettent alors leurs profits à l'abri des folies de la politique. Tant que les citoyens auront peur de leur propre Etat, peur de son mépris pour l'épargne, la propriété et l'investissement rentable, les capitaux continueront de fuir et le pays de s'appauvrir davantage.

- Quatrième explication: l'angle mort de l'économie selon l'expression de Claudia von Werlhof (32).

"Les activités de subsistance orientées vers la production de valeurs d'usage et celles de l'économie du rebut où l'habitant du bidonville réhabilite les caisses et détritus des autres gens pour se fabriquer son abri"

y compris le "Hausfrauisiering", c'est-à-dire la "ménagérisation" ou professionnalisation des tâches domestiques. 
- Cinquième explication: le fonctionnement du marché de travail.

A ce propos, Jacques CHARMES évoque la pluri-activité des salariés et les liens avec l'exode rural:

"Le salarié consacre une partie de son temps, de ses efforts et des moyens de production de son patron (phénomène de la "perruque") pour réaliser une activité indépendante qui peut en venir à représenter, dans ses revenus, une partie plus importante que le salaire. Les revenus primaires de l'activité dite principale peuvent dès lors être trompeurs, même s'ils expliquent pourquoi les migrants ruraux sont toujours aussi nombreux à venir s'entasser dans les villes, attirés qu'ils sont par les revenus informels plus substantiels et de toute façon plus accessibles que les salaires de la main d'oeuvre non qualifiée du secteur moderne" (33).

- Sixième explication: la petite production marchande locale.

Depuis la publication de l'étude du BIT (1972) sur le secteur informel au Kenya, de nombreuses publications empiriques ont vu le jour. Leurs résultats ont été basés sur des critères et indicateurs à répertorier pour essayer d'en saisir la portée. Une revue de la litterature, depuis Keith HART (1971) jusqu'à la veille des années 1990 , permet d'en situer les contours.

\subsection{Critères et indicateurs du secteur informel dans les pays en développement, spécialement l'Afrique.}

\subsubsection{La typologie remontant à Keith HART (1971).}

Les activités considérées comme relevant de l'économie informelle ont été classées selon sept critères, remontant à Keith HART (1971) et repris dans les années 80 par Gerard GRELLET (1986) et par Jacques CHARMES (1987) (34). Dans cette optique, le secteur informel n'appartient ni au secteur moderne ni au secteur traditionnel. Il est plutôt la réalité du "petty commodity production" (35) ou la petite production marchande pour satisfaire les besoins fondamentaux (36) étant donné les problèmes de l'emploi dans le Tiers-monde entre 1960 et 1980.

Cette période enregistre pour l'Afrique, une progression démographique au rythme annuel de 2,5\% (1960-1980) et 3,2\% (1980-1990) pour l'ensemble de la population et de $6 \%$ pour la population urbaine. La croissance de l'emploi offert par le secteur moderne a été beaucoup plus lente, inférieure à $2 \%$ par an. Pour faire face au besoin d'emploi, les urbains non salariés ont trouvé appui dans le secteur informel à raison des critères suivants:

1. la facilité d'entrée; 
2. l'utilisation de ressources locales;

3. la propriété familiale des entreprises;

4. le faible niveau de la production;

5. l'utilisation de techniques à forts coefficients de main-d'oeuvre;

6. un apprentissage effectué hors du système scolaire;

7. des marchés non enregistrés et concurrentiels.

Ces critères établis par K. HART constituent un élément de réflexion sur la démarche de participation au développement dans les années 1970. La distinction entre le secteur informel et le secteur formel convient d'être vérifiée quant aux changements profonds d'un secteur à l'autre, et montrer l'importance des activités de type "petites et moyennes entreprises" informelles (37). En effet, les petites réalisations exploitent des ressources qui autrement ne profiteraient peut-être pas au processus du développement. Elles utilisent les ressources locales et facilitent l'embauche d'un personnel peu qualifié pour lui faire bénéficier d'une formation sur le terrain. Dans une optique d'intégration économique du monde rural, les indicateurs $1,2,5$ et 6 de la typologie peuvent servir de correctifs a la marginalisation du paysan en Afrique. Aussi, en se référant aux critères 3 et 7 , l'on parvient à mobiliser la petite épargne des propriétaires sans passer par le système bancaire mais en investissant dans leur propre affaire. Les projets dits de "basic needs" utilisent les matières premières locales dispersées et ont une propension prononcée à l'épargne et à l'investissement même avec des niveaux de revenus relativement bas si l'on s'appuie aux critères 2 et 4 . Ces projets peuvent s'appuyer sur le critère 5 à savoir "une forte intensité de main-d'oeuvre et une petite taille", ce qui répond, dans certaines dimensions, aux activités d'auto-suffisance interne. De plus, les nombreuses réglementations qui affectent les marchés des pays en développement encouragent souvent les petites entreprises à fonctionner avec des ratios capital/travail non rentables. Les petites entreprises présentent une certaine souplesse étant donné la production de petits lots.

Toutefois, les petites entreprises du secteur informel connaissent certains désavantages vis-à-vis du secteur moderne souvent pourvu de capitaux pour investir. Les petites entreprises informelles sont souvent privées de capitaux, sont abandonnées par les organismes financiers et négligées par les plans de développement. Elles étaient (jusqu'il y a peu) (38) ignorées de la plupart des programmes d'aide financière étrangers. Pour se développer, les activités et les programmes du secteur informel de production comptent sur leurs recettes: elles n'ont pas d'accès au crédit bancaire et institutionnel.

Cette structure de fonctionnement fait des activités informelles issues des enquêtes B.I.T., une réalité pleine de dynamisme et pépinière de petits entrepreneurs, un 
objet de curiosité chez les chercheurs jusqu'à leur révéler d'autres critères de classification complémentaires au schéma de K. HART.

\subsubsection{Les indicateurs précisés par S.V. SETHURAMAN (1976).}

Huit indicateurs sont formules d'abord par S.V. SETHURAMAN (1976) et repris par A.T.M. Nurul AMIN (1982, 1987) à l'issue de ses observations au Bangladesh (39):

1. l'emploi de 10 personnes au plus;

2. la non-application des règles légales et administratives;

3. l'emploi d'aides familiales;

4. l'absence d'horaire ou de jours fixes de travail;

5. l'absence de crédits institutionnels;

6. une formation scolaire des travailleurs inférieurs a 6 ans;

7. l'absence d'énergie mécanique et électrique;

8. le caractère ambulant ou semi permanent de l'activité.

Ces indicateurs soulèvent toutefois des critiques: ils risquent non seulement d'être inefficaces, mais surtout ils sont généraux, descriptifs et peuvent varier. En effet, dans le secteur informel le nombre de personnes employées peut changer suivant l'importance de l'affaire et suivant l'intérêt de chacun des participants. Dès lors en limiter le nombre à 10 personnes convient plus comme simple proposition ou comme constat à partir de l'analyse faite à Dhaka que comme règle. L'emploi d'aides familiales dépend de l'endroit où s'effectue le travail à accomplir et de la disponibilité des membres de famille. La formation scolaire produira des résultats différents selon qu'il s'agit des hommes ou des femmes dans le cas africain. Là-dessus, il serait difficile d'établir des règles absolues car chaque type d'activité peut exiger ses propres structures. Tel est le cas par exemple chez les Bamiléké au Cameroun (40) où il existe une différenciation très nette des occupations selon qu'on est homme ou femme. Les femmes ont leurs propres hiérarchies, leurs propres associations, une forte mobilité sociale et un rôle rituel différent de celui des hommes. Dans la production rurale, les femmes s'occupent des cultures vivrières, des transports, des travaux domestiques mais peuvent exercer d'autres travaux avec les hommes, par exemple le lavage de café; les hommes, de leur côté, s'occupent du défrichement, des cultures arbustives, de la construction de cases et de clôtures, du petit élevage et du commerce. Pour pouvoir financer les mouvements associatifs, les femmes ont une autonomie économique presque totale. 


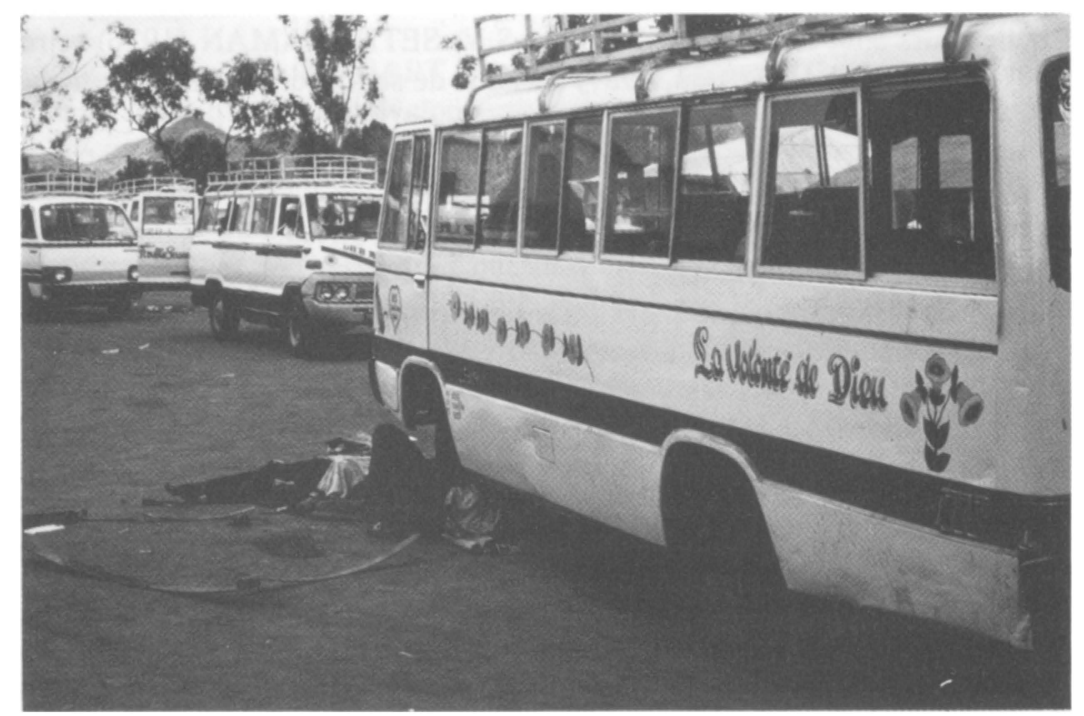

Transport public "informel" au Cameroun

D. Beke ${ }^{\circ}$ 
Les critères de SETHURAMAN et Nurul AMIN, concernant l'absence de crédits institutionnels, l'absence d'horaire ou de jours fixes de travail et le caractère ambulant ou semi-permanent de l'activité informelle relèvent de l'existence même de l'activité. Si elle obtient le crédit de l'Etat, l'activité informelle devient repérable et donc réglementaire et évidente. Le critère en rapport avec la présence d'énergie mécanique et électrique peut varier selon les lieux d'implantation de l'activité et selon les transformations exigées.

L'ensemble des diverses observations empiriques du secteur informel permet d'élaborer d'autres critères, par exemple ceux de formation du capital privé. Pour approfondir la démarche retenons:

\subsubsection{Les indicateurs relevés par Marc LE PAPE (1983).}

Depuis les années 80, la perspective du secteur informel évolue en ce que nous aimerions appeler "économie intégrative". En Afrique, l'effort est de se sortir de la crise - et pas seulement de la crise - étant donné la fragilité économique et la marginalisation de certaines catégories sociales. Les efforts dans le sens d'économie intégrative ne sont pas seulement "urbaines". La préoccupation profonde serait de chercher a travers les petites activités de production des initiatives issues du compromis entre les pratiques économiques officielles et l'économie informelle; cela, en vue d'aider à construire un nouveau modèle où toutes les catégories rurales et urbaines trouvent leur place. Par contre, le secteur rural est peu traité ou presque pas dans la littérature sur le secteur non structuré.

Cette lacune est encore présente dans les descriptions de Marc LE PAPE - datées pourtant des années 1980 - et même dans l'interprétation de Claude de MIRAS (41) par la suite.

Six critères retenus de la classification de M. LE PAPE et à base desquels nous venons de formuler la critique sont:

1. les pourcentages élevés d'actifs non salariés: apprentis, aides familiales, main-d'oeuvre temporaire;

2. le secteur qui se développe à l'écart des réglementations européanisées;

3. une sociabilité particulière, une manière africaine de travailler s'affirmant dans le quotidien artisanal, une progression moderne de petites activités;

4. les établissements sédentaires, le nombre des "actifs de la rue";

5. les petites activités socio-économiques ou les activités visibles de la rue; 
6. l'ensemble du cadre familial large conforme aux mécanismes de solidarité traditionnelle présumé rendre acceptable les très bas salaires ou l'absence de salaire.

Ces indicateurs rendent compte de l'effort africain pour se débrouiller: les equipements techniques sont modiques, les ateliers sont de faible croissance. La part de la solidarité africaine devant l'absence relative de formation de capital et la diversité sectorielle et géographique sont autant d'éléments à observer pour comprendre l'effort déployé en vue de se démarginaliser et donc une démarche d'économie informelle intégrative.

Ces éléments ne se retrouvent pas seulement dans les milieux urbains mais aussi dans les milieux ruraux. Et pour preuve de leur existence dans les milieux ruraux, nous pouvons recourir aux critères avancés par Philippe HUGON (1985) dans son analyse de la dépendance alimentaire des villes en Afrique (42) et spécialement à Madagascar.

\subsubsection{Les critères avancés par Philippe HUGON (1985).}

Il existe une certaine interdépendance des milieux urbains et des milieux ruraux dans la production agro-alimentaire. Les enquêtes de $\mathrm{Ph}$. HUGON sur la consommation rizicole à Madagascar étayent ce point de vue. Mais plus proche de nous par rapport aux espaces ruraux, la production de légumes appelés "Mboga" à Bukavu (Est du Zaïre) permet aux paysans d'entrer en relation avec le milieu urbain par le biais du marché informel et du marché formel. Cette production agricole issue des cultures maraîchères de "Bukavu rural" est commercialisée tout au long de l'année et correspond aux indicateurs d'économie informelle retrouvée dans les pages écrites par le "spécialiste" de petite production marchande urbaine.

\section{Il s'agit de:}

1. activités de production agricole à petite échelle;

2. activités organisées selon des relations non salariales;

3. techniques simples;

4. capital avancé faible;

5. circulation monétaire et jeu de prix;

6. produit alimentaire $=$ marchandise.

Ainsi se révèle-t-il, par rapport à la production rurale, un autre aspect qui va déterminer le champ de l'enquête sur le terrain. Les mboga permettent d'aborder l'aspect productif du secteur informel du point de vue agricole étant donné le revenu rural généré par ces produits et la diversification agricole qu'ils entraînent. 
L'angle productif de l'économie informelle vise, bien entendu, le revenu rural comme réponse grâce à laquelle les populations se défendent et se protègent, mais aussi se dynamisent et innovent dans le processus de mise en relation avec le secteur moderne. Bref, une réponse en vue de satisfaire les besoins non assouvis par le secteur officiel de l'économie au fur et à mesure que ce dernier s'est dégradé (43).

\section{CONCLUSION.}

L'image courante projetée par la littérature semble réduire l'économie informelle aux transactions économiques rebelles à l'enregistrement (44) ou à l'imposition par les autorités. Les exemples portent sur des revenus perçus mais non déclarés aux autorités de l'administration publique. Dès lors comment les études examinées observent-elles la réalité de l'économie informelle?

Analysée de l'Occident, l'économie informelle concerne les activités productives souterraines, irrégulières et dissimulées: il s'agit des productions informelles par convention (Angleterre, Allemagne) et par manque de moyen de les inclure dans le Produit National Brut. Elle incarne également les productions difficilement Évaluables: ce sont les activités sans nécessité économique et le travail pour soi; elles sont reconnues mais posent des problèmes d'évaluation. Les activités criminelles relèvent aussi du secteur non marchand comptabilisable mais qui échappe au fisc et aux statistiques. Evaluées ou non, les activités informelles en Occident ne sont pas sans impact (fût-ce provisoire) sur l'ensemble de la production nationale.

Du point de vue soviétique et de l'Europe de l'Est, l'économie informelle serait une tentative de revolte contre la socialisation jugée abusive par l'individu d'une part et un effort de défendre ses droits en milieu économique d'autre part. Aussi, une imbrication des rapports du jeu économique entraîne les diverses tentatives d'inspiration sociale visant à corriger les vices de régimes politiques. Ce dernier point serait, à notre avis, commun au secteur informel de l'Europe de l'Est et de l'Afrique, au cours des années 80.

Dans les pays en voie de développement, spécialement l'Afrique, la réalité de l'économie informelle s'observe, de notre point de vue, par rapport à trois periodes:

a. a partir des années 60, un point de vue socio-anthropologique justifie les diverses tentatives de redistribution et de socialisation: le recours à la conscience 
collective. Goran HYDEN situera dans cette logique son "Économie d'affection" (45) charriant avec elle les impératifs culturels et moraux auxquels se heurteraient les choix économiques. Comme fin idéologique, sa caractéristique principale serait la logique sociale de réciprocité et la philosophie de gestion trans-tribale de la nation (46);

b. par rapport au progrès de l'industrialisation dans les années 70 , toute activité économique en dehors des structures institutionnelles serait parasite de l'économie officielle. Le concept de Goran HYDEN semble déjà présent dans les approches (47).

Le secteur informel est désigné comme "secteur non structuré" malgré sa façon de s'organiser et fonctionnerait dans l'illégalité qui pervertit le marché et l'Etat. Quoiqu'il en soit, la nature de l'économie informelle au milieu des années 70 apparaît, pour les uns, comme pleine de dynamisme et pépinière de petits entrepreneurs. Ceux qui y prospèrent, il faut les aider. Bref, prévoir un programme d'aide pour cela. Pour d'autres analystes, il s'agit d'une économie subordonnée au secteur moderne. Elle permet à l'accumulation de se réaliser dans de bonnes conditions parce que la main d'oeuvre est disponible.

c. par la suite, vers la fin des années 80 , la perspective évolue en une économie sociale qui peut s'articuler avec l'économie nationale étant donné la réalité de dualisme, d'exclusion mais aussi des formes d'intégration qui régissent les marchés nationaux. Le secteur informel serait, en cette période, un concept global à ne pas réduire à sa dimension purement économique. Il reposerait sur une diversité interne de ses activités marchandes et non marchandes dans le fonctionnement de l'économie africaine.

Comme tentative créatrice et souvent efficace de répondre aux défis d'une société malmenée, le secteur informel se présente finalement comme petite activité de production et de consommation. Ses activités productives sont percues par rapport à la réalité du "petty commodity production" pour satisfaire les besoins fondamentaux. Il s'agit de construire son mode de vie en fonction des ressources localement installées, des techniques maîtrisables et de l'identité culturelle. Bref, reconnaître à travers cette autre forme d'invention et de production, la force d'un modele qui met en valeur un rapport social.

L'absence de cette économie informelle dans les comptes nationaux n'est pas uniquement conventionnelle comme en Angleterre et en Allemagne; elle n'est pas due nécessairement au manque de moyen d'inclure le secteur informel productif dans les comptes sous prétexte qu'il est souterrain, irrégulier ou dissimulé. Le fond 
du problème réside dans le fait que les activités productives de l'Economie informelle ne sont pas reprises par les autorités des pays en développement par peur d'en endosser la responsabilité en matière de crédit financier. Autour de ce manque de volonté politique surgissent les questions de dualisme, d'exclusion et d'intégration. C'est pourquoi nous appuyons l'économie informelle comme étant la tentative du secteur oublié d'apporter, par sa production, une réponse défensive et protectrice mais aussi dynamique et innovatrice au processus de mise en relation avec le secteur moderne; cela, en vue de satisfaire les besoins non assouvis par le secteur officiel de l'Economie, en vue aussi d'utiliser des ressources non employées et donc de participer au développement économique à différents échelons emboîtés l'un dans l'autre à savoir le local, le régional, le national et l'international.

NOTES

1. Michel MOUGEOT, "Le rapport Dupeyroux ou comment révéler les transactions cachées", in Revue d'Economie Politique, $n^{\circ} 3,94 \mathrm{e}$ anné, mai-juin 1984, p. 407-412.

Et tout récemment: Catherine COQUERY-VIDROVICH, "L'informel dans les villes africaines: essai d'analyse historique et sociale" in Tiers-Monde: L'informel en question? (ouvrage collectif), L'Harmattan, 1991, 385 p., P. 171: "Il paraît clair qu'en Afrique comme ailleurs des formes de travail et de vie 'non contrôlées' ont existé en ville depuis longtemps. Le fait, on va le voir dans un instant, est patent à l'époque coloniale. Mais il est apparemment possible de remonter au paravant, au moins au XVIle siecle en Afrique de l'Ouest, d'après les sources disponibles et explorées jusqu'à présent." Le travail a été en partie effectué par le chercheur anglophone John ILIFFE, The African Poor. A History, Cambridge University Press, 1987.

2. Un financier italien qui proposa et fit accepter par le cardinal MAZARIN de France (1562) un projet d'emprunt public, une sorte de rente viagère: David WEIR, "Tontines, Public Finance and Revolution in France and England, 1688-1789", in The Journal of Economic History, Vol. XLIX, 1, mars 1989, p. 95-124.

3. Xavier GREFFE, "L'économie non officielle", in Problèmes économiques, $n^{*} 1771,28$ avril 1982 , p. $3-14$, p. 3.

4. Guy QUADEN, Politique économique, éd. Labor, 1990, 342 p., p. 65, "Le problème de l'économie souterraine n'est pas nouveau".

5. Pierre PESTIEAU, L'économie souterraine, Hachette, 1989, 320 p., p. 155-159.

6. Stephen SMITH, "European perspectives on the Shadow Economy", in European Economic Review 33 (1989), p. 589-596. 
7. Ivan ILLICH, Le genre vernaculaire, Seuil, 1983, 252 p.

8. Marie-Annick BARTHE, L'économie cachée, 1988, p. 12.

9. André GORZ, Métamorphoses du travail. Quête du sens. Critique de la raison économique, éd. Galilée, 1988, 304 p., p. 173-186, p. 191-205.

10. Selon les auteurs, "autonome" s'applique tantôt à l'auto-production artisanale, tantôt à n'importe quelle forme "alternative", autogérée ou autodéterminée d'activité marchande. Pour André GORZ, op. cit., 1988, p. 208, il s'agit d'activité sans nécessité ni utilité économique aucune: l'activité serait elle-même sa propre fin. Elle n'est pas payée mais crée un bien-être, nous semble-t-il, en augmentant l'autonomie des gens.

11. Marcel DRACH \& Beranrd TOBOUL, "L'économie parallèle allemande", in Le Monde Diplomatique, avril 1989, p. 7.

12. Jean Dominique LAFAY, "L'économie de la corruption", in Analyses de la S.E.D.E.I.S., $n^{\circ} 74$, mars 1990 , p. 62-66.

13. Stuart HENRY, "The Working unemployed : perspective on the informal economy and unemployment", in The Sociological Review, Vol. XXX, 3, Aug. 1982, p. 460-477.

Stephen SMITH \& Suzanne WIED-NEBBELING, The Shadow economy in Britain and Germany, Anglo-German Foundation, 1986, 102 p., p. 3-4.

Jean-Charles WILLARD, "L'économie souterraine dans les comptes nationaux", in Economie et Statistiques, nov. 1989, in Problèmes économiques, $n^{\circ} 2160,31$ janv. 1990, p.3.

14. "tout ce qui relève de la recherche du gain privé, plus tout ce qui est en contravention avec la loi". Gregory GROSSMAN, "La seconde économie et la planification économique soviétique", in Revue d'Etudes Comparatives Est-Ouest, Vol. XII, 2, juin 1981, p. 1-24; Pierre PESTIEAU, op. cit., 1989, p. 167.

15. Krzysztof STARZEC, "L'économie polonaise vue à travers des circuits parallèles", in Consommation - Revue de Socio-Economie, $n^{*} 4$, Oct. déc. 1983, p. 55-94.

Rozine KLATZMANN, Le travail noir, coll. "Que sais-je?", P.U.F., 1982, 128 p., p. 70-71.

16. Gregory GROSSMAN, op. cit., p. 5 .

17. O.V. OSIPENKO, I.G. KOZLOV, "What Is It That Casts a Shadow?", in Problems of Economics. A Joumal of Translations, vol. XXXII, 7, nov. 1989, p. 29-42.

18. Ivan ILLICH, Le genre vernaculaire. Seuil, 1983, 252 p., p. 26-27. 
19. Agota DEZSNENYI-GUEULLETTE, "L'économie parallèle à l'Est : le cas hongrois", in Revue d'Etudes comparatives Est-Ouest, vol. XII, $n^{\circ} 2$, juin 1981, p. 25-39.

20. Marc LE PAPE, "De l' "indigène" à l' "informel", 1955-1982, in Cahiers d'Etudes Africaines, 89-90 à 92, 1983, p. 189-197.

21. La préhistoire du secteur informel aurait été marquée en premier lieu par les travaux de l'East Royal Commission de 1953-1955 au Kenya; en second lieu par la grande conférence de Kericho en 1966 pour prendre conscience de la nécessité de transformer l'économie rurale traditionnelle étant donné la dure arithmétique du chômage: Kenneth KING, "La recherche, la politique et le secteur informel: l'experience africaine", in Nouvelles Approches du secteur informel, OCDE, 1990, p. 155-177, p. 158.

22. Philippe HUGON, "Approches pour l'étude du secteur informel (dans le contexte africain), in Nouvelles Approches du secteur informel, OCDE, 1990, p. 81-88.

23. K. HART 1971, S.V. SETHURAMAN 1976, J. CHARMES 1983, 1987, 1990: 3, G. GRELLET, 1986.

24. Lisa R. PEATTIE, "Anthropological Perspectives on the Concepts of Dualism the Informal Sector, and Marginality in Developing Urban Economies", in International Regional Science Review, Spring 1980, p. 70-88, p. 23.

25. J.F. LAE, "Le travail au noir, vestibule de l'emploi", in Sociologie du travail, XXXII, 1, 1990: p. 23-37.

26. Jocelyne GAUDIN et Michel SCHIRAY, "L'économie cachée en France: état du débat et bilan des travaux" in Revue économique, vol. 35, 4, 1984, p. 691-731.

Ignacy SACHS, "La crise, le progrès technique et l'économie cachée" in Communautés. Archives de Sciences Sociales de la Coopération et du Développement, $n^{\circ} 67$, janvier-mars 1984, p. 110-118.

27. Jacques CHARMES, "Comment mesurer la contribution du secteur non structuré à la production nationale dans les pays du Tiers-Monde?" in The Review of Income and Wealth. Journal of International Association for Research, Income and Wealth, Series 29, 1983 , p. $429-444$.

28. Stuart HENRY, "The working unemployed: perspectives on the informal economy and unemployment", in The Sociological Review, Vol XXX, 3, 1982, p. 460-477, p. 463.

29. A. CHANDAVARKAR, "The informal Sector: Empty Box or Portemanteau Concept? (A comment)" , in World Development 16 (10), 1988, p. 1259-1261.

30. Xavier GREFFE, "L'économie non officielle", in Consommation, Revue de socio-économie, juillet $n^{\circ} 3,1981$, et in Problèmes économiques $n^{\circ} 1771,28$ avril 1982, p. 3-14. 
31. Guy SORMAN, La nouvelle richesse des nations, éd. Fayard, 1987, 380 p.: "Economie informelle", p. 54-56, p. 111-114, p. 206.

32. Ivan ILLICH, op. cit., 1983, p. 43 et p. 73. Divers concepts ont été crées pour désigner l'adhésion normative à un code consacré à la survie et aux postulats de la rareté. Ainsi pour l'anthropologie rurale, le concept correspond à "l'éthique de la subsistance" et selon le concept d' E.P. THOMPSON, ce serait de "l'économie morale". ILLICH retient dans cette catégorie l'expression de la sociologue allemande Claudia WERLHOF spécialement célèbre pour ses études sur la professionnalisation des tâches domestiques. Voir André GORZ, op. cit., 1988, p. 195.

33. Jacques CHARMES, "Une revue critique des concepts, définitions et recherche sur le secteur informel, in Nouvelles Approches du Secteur Informel, Séminaire OCDE, 1990, p. 11-15, Chap. 1, p. 49.

34. Gérard GRELLET, Structures et stratégies du développement économique, P.U.F., 1986, 451 p., p. 344-349, il définit le secteur informel et la position des experts du Bureau International du Travail dans leur rapport sur l'emploi au Kenya. Il se réfère à K. HART, "Informal opportunities and Urban Employment in Ghana", Journal of Modern African Studies, 1973.

Jacques CHARMES, "Débat actuel sur le secteur informel", in Revue Tiers-Monde, n`112, t. XXVIII, oct.-déc. 1987, p. 855-875.

35. Caroline O. MOSER, "Informal Sector or Petty Commodity Production: dualism or dependence in Urban Development?" in World Development, vol. 6, n' 9/10, 1978, p. 1041-1064.

36. Bernard FOUNOU-TCHUIGOUA, "L'approche par les besoins essentiels, une théorie nouvelle de développement pour la décennie 1980 ? in Africa Development, $n^{\circ} 3,1980, p$. 37-67.

37. MARSDEN, K., "Créer un environment favorable aux petites entreprises", in Finances et Dévelopment, $n^{\bullet} 4$, déc. 1981 , p. 33-36.

38. En effet, au nom de l'efficacité, les Organisations de développement commencent à se réconcilier avec les entreprises et les institutions publiques au point de constituer des réseaux, recevoir des appuis bancaires et conférer aux projets de développement un caractère productif. Les ONG acceptent ainsi d'oeuvrer entre l'activité économique et l'activité caritative pour une meilleure reconnaissance de leur action sur le terrain: Eric FOTTORINO, "L'aide au développement, du romantisme au réalisme", in Le Monde, mardi 22 janvier 1991, p. 33.

39. S.V.SETHURAMAN, "Le secteur urbain non structuré: concept, mesure et action", in Revue internationale du Travail, Vol. 114, $\mathrm{n}^{\circ}$ 1, juillet-aôut 1976, p. 79-92. 
A.T.M. Nurul AMIN, "Le rôle du secteur non structuré :dans le développement économique. Observations faites à Dhaka, Bangladesh", in Revue internationale du Travail, Vol. 126, $\mathrm{n}^{\circ}$ 5, sept.-oct. 1987, p. 687-700.

40. Gabriel GOSSELIN, Développement et tradition dans les sociétés rurales Africaines, B.I.T., 1970, 343 p., p. 45-89, souligne les structures sociales particulières des Bamiléké et leur impact sur les principes fondamentaux de financement du crédit.

41. Claude de MIRAS, "De l'accumulation de capital dans le secteur informel", in Cahiers de Sciences Humaines, 23 (1), 1987, p. 49-74.

42. Philippe HUGON, "Dépendance alimentaire et urbanisation en Afrique: un essai d'analyse méso-dynamique en termes de filières", in Nourrir les villes en Afrique sub-saharienne, éd. L'Harmattan, 1985, 421 p., p. 23-46.

43. MUHEME BAGALWA B.G., "De toekomst van het "mboga-"netwerk, als een informeel-ekonomisch antwoord op de ekonomische integratie van de Zaïrese plattelandsbevolking", in Noord-Zuid Cahier. De Informele Economie en de Derde Wereld, Wereldwijd, jaargang $15, \mathrm{n}^{\circ} 4$, december 1990 , p. 3-13.

44. Le Congrès des économistes belges de langue française tenu à Charleroi les 15 et 16 janvier 1987 est du même avis. Il manque de renseignements sur l'importance de l'économie souterraine: Vincent GINSBURG et Pierre PESTIEAU, L'Economie Informelle. Fraude fiscale, travail au noir et autres activités non déclarées. Coll. Economie 2000, éd. Labor, Bruxelles, 1987, 189 p.

45. Goran HYDEN, No shortcuts to progress. African development management in perspective, London, 1983, 223 p.: p. 1-29.

46. René LEMARCHAND, "African Peasantries. Reciprocity and the Market. The Economy of Affection Reconsidered, in Cahiers d'études africaines, XXIX (1), 113, 1989: 33-67, p. 35 .

47. Janet MacGAFFEY, Entrepreneurs and Parasites. The struggle for indigenous capitalism in Zaire, Cambridge University Press, 1987, 241 p. 


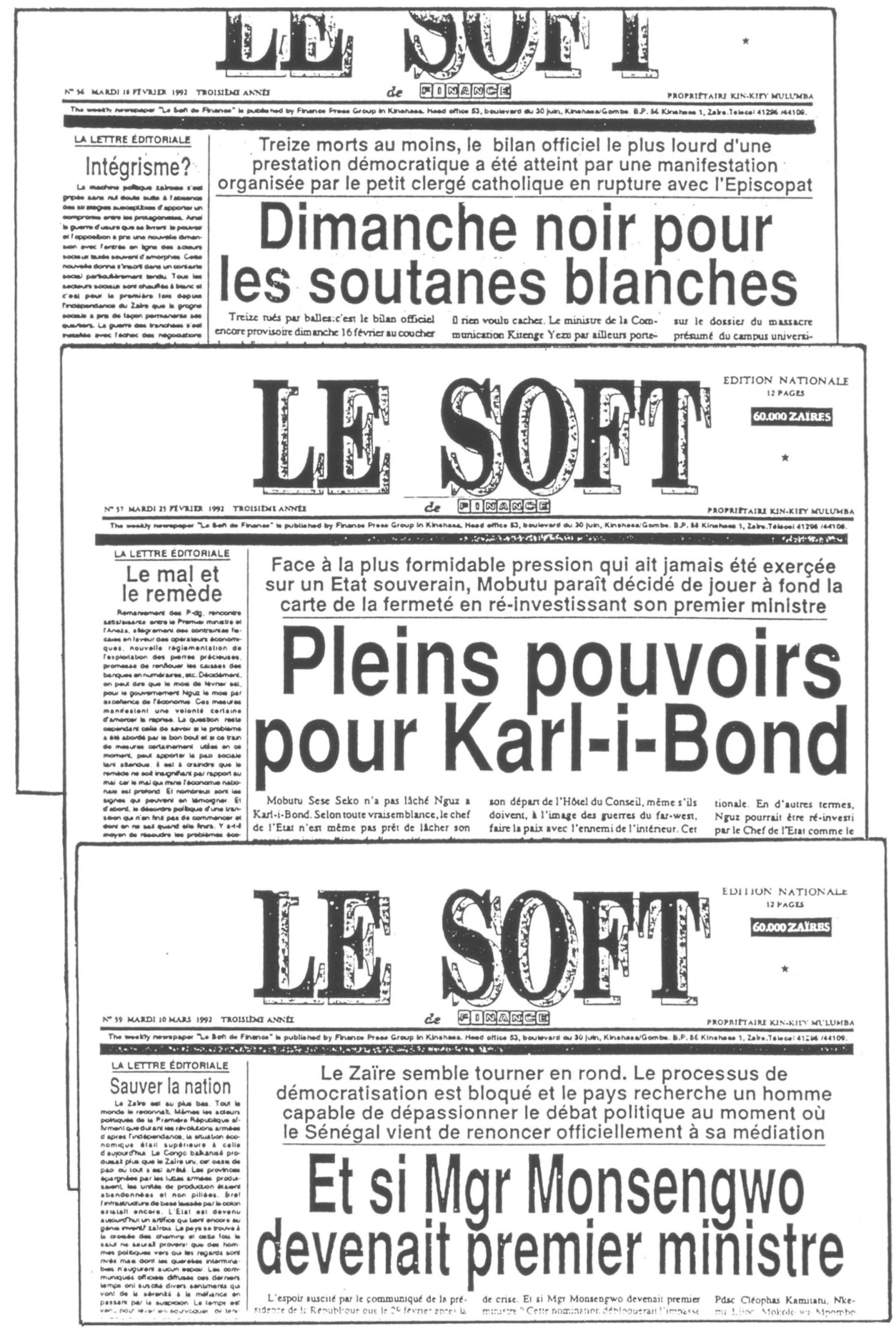

